\title{
Teoría del caos y método científico
}

\author{
Coppo, J.A.
}

Profesor de "Epistemología" y "Metodología de la Investigación” en cursos de posgrado de la Facultad de Ciencias Veterinarias, UNNE, Sargento Cabral 2139, Corrientes (3400), Argentina. Tel/fax 03783-425753. E-mail: jcoppo@vet.unne.edu.ar.

\begin{abstract}
Resumen
Coppo, J.A.: Teoría del caos y método científico. Rev. vet. 21: 2, 157-167, 2010. Se efectúa una revisión bibliográfica sobre la relación epistemológica entre la ciencia y el caos. En el pasado, el mecanicismo había postulado un universo ordenado y continuo formado por partes separadas (reduccionismo) que respondían a una causa (causalidad) y podían provocar efectos predecibles (predictibilidad). En las últimas décadas surgió la concepción de los sistemas complejos, muchos de ellos desordenados, a-periódicos y disipadores de energía, cuyas partes no se conciben sin el todo (holismo), lo cual generó un cambio en la filosofía de la ciencia y justificó su comprensión a través de la teoría del caos. Los sistemas caóticos son muy sensibles a las condiciones iniciales (determinismo) e impredecibles a largo plazo. Se narra una breve historia sobre esta nueva metodología de investigación científica y se detallan sus componentes, tales como fractales, atractores, ruidos, espacios de fases y dimensiones. Con el advenimiento de la informática nació la matemática del caos, que junto a la lógica difusa, permitieron construir modelos experimentales (simuladores) con los cuales se obtuvieron nuevos conocimientos y predicciones en los campos de la fisiología, patología, biología, física, meteorología, economía y otros. En fisiología se elucidaron fenómenos concernientes al biorritmo del sistema nervioso, automatismo cardíaco, dinámica circulatoria, homeostasis del medio interno, adaptabilidad pulmonar, ritmo circadiano, retroacciones hormonales, actividades enzimáticas, eficiencia inmunológica, percepción olfatoria, cognición, conducta y cambios adaptativos. La teoría del caos ha motivado un cambio de paradigma y se ha convertido en un método para generar conocimientos científicos en el área de los sistemas complejos inestables.
\end{abstract}

Palabras clave: caos determinístico, ciencia, sistemas complejos, predictibilidad.

\begin{abstract}
Coppo, J.A.: Chaos theory and scientific method. Rev. vet. 21: 2, 157-167, 2010. A bibliographical review on the epistemological relationship between science and chaos is presented. In the past, mechanicism had postulated an orderly and continuous universe formed by separated parts (reductionism) which responded to a cause (causality) and might generate predictable effects (predictability). The conception of complex systems, many of them disordered, aperiodical, and energy dissipators, appeared in the last decades. The parts of these systems are not conceived without the whole (holism); this concept generated a change in the philosophy of science and justified its understanding through the chaos theory. Chaotic systems are very sensitive to the initial conditions (determinism) and are unpredictables to a long term. A brief history on this new scientific investigation methodology is narrated. The chaos components, such as fractals, attractors, noises, phase-spaces, and dimensions, are described. Mathematics of the chaos was born due to computer science's development. This mathematics, together with diffuse logic, allowed to build experimental models (simulators) by means of which new knowledge and predictions in the fields of physiology, pathology, biology, physics, meteorology, economy and others, were obtained. In physiology, phenomena concerning the biorhythm of the nervous system, heart automatism, circulatory dynamics, internal environment homeostasis, lung adaptability, circadian rhythm, hormonal retroactions, enzymatic activities, immunologic efficiency, olfactory perception, cognition, behavior and adaptive changes, were elucidated. Chaos theory has motivated a paradigm change and it has become a method to generate scientific knowledge in the area of unstable complex systems.
\end{abstract}

Key words: deterministic chaos, science, complex systems, predictability. 


\section{Introducción}

"por un clavo se perdió la herradura por una herradura se perdió el caballo por un caballo se perdió el jinete por un jinete se perdió la batalla por una batalla se perdió el reino"

En el acápite del artículo se consignó un poema folclórico británico cuya conclusión podría ser "por un clavo se perdió el reino”. Debería inferirse que el desconocido autor de esta trova debió ser un experto en la teoría del caos, pues la secuencia utilizada enuncia con elocuencia que por algo tan insignificante como un clavo se llegue al extremo de perder un reino. Tal magnificación puede parangonarse con el "efecto mariposa".

El "efecto mariposa" es una alegoría que, en el contexto de la teoría del caos, remarca la enorme importancia de la sensibilidad a las condiciones iniciales (Figura 1). El concepto implica que insignificantes variaciones de las condiciones iniciales de un determinado sistema caótico, pueden provocar respuestas completamente diferentes. Así, un efecto colosal podría haber sido causado por la amplificación de una minúscula perturbación inicial $^{9}$.

Tal interrelación causa-efecto ocurre más frecuentemente de lo que imaginamos. En sistemas complejos tales como el organismo humano o animal, el estado del tiempo o la bolsa de valores, el "efecto mariposa" indica que será muy difícil predecir con seguridad sus comportamientos en un determinado lapso. Al tratar de simular algún sistema mediante modelos finitos, inevitablemente se excluirá información acerca del sistema y/o de sus componentes relacionados, error que será magnificado en cada unidad de tiempo simulada, hasta que el error resultante llega a exceder el ciento por ciento. O sea: aunque se conozca el modelo, pasado cierto tiempo habrá una divergencia importante con la realidad. En efecto, ello fue lo que le sucedió accidentalmente a Edward Lorenz (1917-2008), científico norteamericano que efectuó trascendentes aportes a la teoría del caos, introduciendo los conceptos de "efecto mariposa" y "atractor extraño" 11,22.

Lorenz (Figura 2) era físico, matemático y meteorólogo del Institu-

to de Tecnología de Massachusetts. Aprovechando la capacidad de cálculo de una gran computadora de la institución, en 1961 construyó un modelo para predecir el tiempo atmosférico mediante ecuaciones que expresaban las relaciones entre temperatura, presión, velocidad del viento, humedad y otros datos, empleando un programa (simulador) que representaba gráficamente las distintas variables, mediante curvas. Un día introdujo en la computadora los datos de las series del día

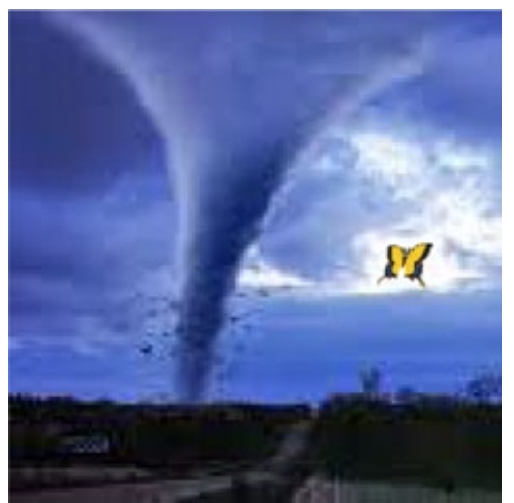

Figura 1. "El aleteo de una mariposa en China puede desatar un tornado en Argentina".

anterior (expresados con seis decimales), aunque para ahorrar tiempo los escribió con tres decimales, esperando un resultado igual o similar al anterior. Grande fue su sorpresa cuando comprobó que los resultados eran semejantes en el corto plazo, pero se tornaban totalmente diferentes en el mediano y largo plazo. Observó que a partir del primer valle de la curva, ésta comenzaba a separarse y rápidamente ambas curvas perdían cualquier similitud. El motivo era que su programa trabajaba con millonésimas y Lorenz había redondeado los datos a milésimas. Pese a ser un pequeño cambio numérico, ese input ligeramente distinto tuvo efectos sorprendentes en el output. Este fenómeno es conocido como dependencia sensitiva de las condiciones iniciales: una pequeña variación puede cambiar drásticamente el comportamiento "a larga distancia" de un sistema ("efecto mariposa") 14, 22 .

Se afirma que el "efecto mariposa" no es accidental, sino necesario. De no producirse, los ciclos meteorológicos obtenidos con estos modelos informáticos serían iguales, rígidos, sin reproducir la variación climática real. El tiempo atmosférico, además de ser un sistema dinámico, es muy sensible a los cambios en las variables iniciales, por lo cual su estudio debe abordarse desde la matemática caótica. No obstante, la precisión de las predicciones meteorológicas es relativa y los eventos anunciados tienen poco significado sin una descripción detallada de los criterios empleados para juzgar la exactitud de una predicción. Hoy en día es común atribuirles una precisión del $80-85 \%$ para un plazo de 24 horas ${ }^{13-15}$.

\section{Nuevas ideas}

Hace menos de 20 años se ha estado produciendo una revolución en el mundo de las ideas científicas que no ha sido conocida por el público en general. Para describir y entender una multitud de fenómenos inherentes a diversas ramas del conocimiento, han surgido 
conceptos nuevos tales como la teoría del caos y los fractales. En campos como biología, medicina, física, matemáticas y otros, ocurrieron situaciones que al ser tratadas con los procedimientos convencionales no pudieron ser explicadas satisfactoriamente. Sólo con el advenimiento de las ideas nuevas fue posible progresar en el conocimiento de hechos antes no comprendidos 5 .

El concepto de caos a menudo puede crear una idea negativa, una visión de desorden en el cual las cosas no funcionan bien en un mundo donde lo correcto es precisamente el orden. El término caos alude a una interconexión subyacente que se manifiesta en acontecimientos que aparecen como aleatorios y desordenados. El mecanicismo newtoniano nos había enseñado que en el universo existía un orden total y continuo, una serie de bloques mecánicos en interrelación, partes separadas de la realidad (reduccionismo) que respondían a una causa y provocaban un efecto predecible. El paradigma del caos insta a ver la realidad como un todo en donde cualquier factor, por pequeño que parezca, puede afectar el comportamiento y la evolución de la naturaleza. Es "encontrar el orden en el desorden" 31.

Hoy la comunidad científica dispone de conocimientos acerca del caos, desorden, fractales, aperiodicidad y otros, que operan como herramientas para explicar muchos fenómenos de la naturaleza y/o experimentos controlados de laboratorio, caracterizados por tener un comportamiento que no puede ser descrito por leyes matemáticas sencillas. El descubrimiento del caos determinista ha forzado un cambio sustancial en la filosofía de la ciencia: por una parte establece límites a nuestra capacidad para predecir un comportamiento y por otra abre un nuevo espacio para comprender muchos fenómenos aleatorios que suceden en varios campos del conocimiento, caracterizados por su devenir desordenado, como el ritmo cardíaco o la evolución de una epidemia ${ }^{28,31}$.

\section{Teoría del caos}

La teoría del caos es un modelo teórico que intenta explicar el proceder de sistemas dinámicos que en principio parecen desarrollarse aleatoriamente, como por ejemplo el comportamiento del tiempo atmosférico, proponiendo una nueva manera de estudiar y comprender la realidad. De este modo la teoría de caos intenta otorgar una explicación tanto para modelos físicos y matemáticos, como para gran parte de los fenómenos naturales, desde la propagación de un incendio hasta la evolución de una sociedad. Por ello es comprensible que las leyes de la teoría del caos sean útiles para ser aplicadas en múltiples áreas de la ciencia, sobre todo en los llamados movimientos caóticos, entre los cuales encontramos los movimientos fisiológicos de las partículas de los fluidos y la homeostasis del medio interno. Tales sistemas son deterministas, es decir; su comportamiento está completamente determinado por sus condiciones iniciales ${ }^{31}$.
Podemos considerar el caos como la ciencia de la naturaleza global de los sistemas. Defiende el comportamiento universal de lo complicado. El caos se ha convertido de una teoría a un método, a una forma de hacer ciencia. Caos no es inestabilidad: un sistema caótico sería estable si un tipo particular de irregularidad persistiera frente a pequeñas perturbaciones, teniendo en consideración la dependencia sensitiva de las condiciones iniciales ${ }^{17}$.

La teoría del caos puede ser definida como el estudio de la conducta aperiódica en sistemas determinísticos no lineales. Un sistema determinístico no lineal consiste en elementos que tienen influencias no lineales entre sí. El sistema es dinámico si cambia en el tiempo. El estado de un sistema dinámico en un cierto momento puede ser descrito por cierto número de variables y puede ser representado por un punto en un espacio de fase de determinadas dimensiones. La evolución en el tiempo consiste en una serie de puntos que forman una trayectoria en el espacio de estados. Cuando el tiempo tiende a infinito, la trayectoria sólo ocupará un subespacio del espacio de estados, denominado atractor ${ }^{1}$.

El caos sería un punto intermedio entre lo estrictamente determinístico y lo aleatorio. Se refiere a fluctuaciones irregulares, las cuales son descritas por ecuaciones deterministas, diferentes de las fluctuaciones que obedecen a causas aleatorias. Las principales características de los sistemas caóticos es que son impredecibles en períodos amplios de tiempo y muy sensibles a las condiciones iniciales del sistema. Una vez iniciado con valores específicos, el futuro sistema podría desarrollarse de forma totalmente diferente si comenzase bajo condiciones ligeramente diferentes. La importancia de este hecho para los sistemas biológicos es que desórdenes muy similares podrían estar determinados por muy pocos y simples factores que podrían llegar a ser conocidos ${ }^{1,11}$.

\section{Historia de la teoría del caos}

Esta teoría tuvo origen a principios del siglo $\mathrm{XX}$, cuando los físicos creían que ya no quedaba mucho por descubrir dentro de su campo de estudio. De hecho, eran sólo tres los interrogantes que quedaban por dilucidar: (1) la explicación de la órbita irregular del planeta Mercurio, (2) la discrepancia entre la teoría y la cantidad de energía que libera un agujero negro y (3) el efecto de un tercer cuerpo en el movimiento de otros dos. A medida que los científicos intentaban dar con estas explicaciones, comenzaron a nacer distintas teorías: del primer problema surgió la teoría de la relatividad, del segundo la teoría cuántica y del tercero la teoría del caos ${ }^{18}$.

En términos generales, esta última hace alusión a aquella tendencia general al desorden en la naturaleza, que se pone en evidencia cuando se rompe un vidrio o se cae un vaso de agua. Es importante destacar que este desorden o caos no implica confusión, por el contrario, los sistemas caóticos tienen como característica 
una gran adaptación al cambio, y por ende una gran estabilidad. Un buen ejemplo del desorden mencionado lo constituye el tirar una piedra a un río. Una vez realizada esta acción el cauce del río no se ve interrumpido. $\mathrm{Si}$ el río fuese un sistema ordenado, en el que cada partícula tuviera una trayectoria fija, entonces la piedra hubiera afectado este orden 18,24 .

James Maxwell (1831-1879), pionero del electromagnetismo, fue una de las primeras personas en entender lo que hoy llamamos "caos determinístico" al reconocer la importancia de los sistemas que dependen de las condiciones iniciales. Henri Poincaré (1854-1912) adhirió a las ideas de Maxwell, llegando a la conclusión que no es posible predecir con exactitud la evolución futura de algunos sistemas porque las condiciones iniciales sólo se conocen de manera aproximada, situación que provoca perturbaciones impredecibles en su comportamiento. Decía Poincaré: “¿porqué es muy natural que la gente rece para que llueva, pero se consideraría ridículo rezar

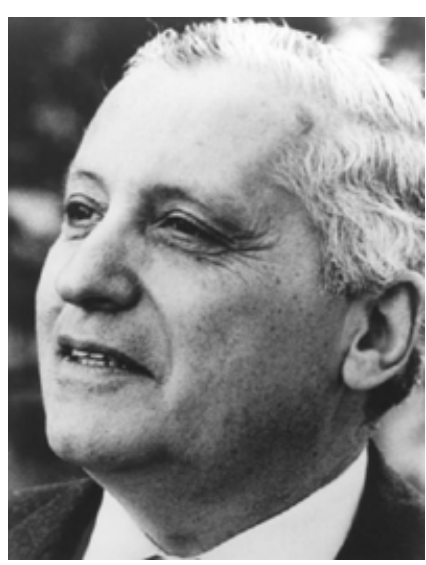

Figura 3. Ilya Prigogine, Premio Nobel de Química 1977, postuló que los sistemas vivientes tienden hacia el orden (ganancia de entropía). turas fractales en secuencias de errores en la transmisión computacional de datos, en las crecidas del Nilo, en la forma de las nubes y en el contorno de las costas. Mitchell Feigenbaum (1976) aplicó el caos en la percepción y planteó que "para entender cómo la mente entresaca algo del caos de la percepción, hay que entender de qué manera el desorden produce universalidad". Robert Shaw (1977) descubrió la relación entre el caos, los atractores, y la teoría de la información fundada en la entropía, postulando que "el caos es la creación de la información” (sin caos no hay sorpresa, y por ende no hay información). Raymond Ideker (1983) aplicó la teoría del caos para efectuar predicciones exitosas de las alteraciones del ritmo cardíaco (fibrilación, disrritmias, muerte súbita infantil). Arnold Mandell (1988) descubrió comportamiento caótico en actividades enzimáticas del cerebro y postuló que los sistemas periódicos (ciclos estables) son monótonos (vacíos de información, poco adaptativos), en tanto que los sistemas biológicos son de tipo fractal, con ampara que haya un eclipse?" Henry Adams (1838-1918) estudió el caos y la dispersión de la energía, creando los aforismos "mientras el orden es el sueño del hombre, el caos es la ley de la naturaleza" y "mientras que el orden crea hábito, el caos con frecuencia crea vida". Ilya Prigogine (1917-2003), físico ruso, estudió el azar, el caos, la incertidumbre y el no-equilibrio, desde una óptica que negaba toda concepción determinista del universo. En 1977 Prigogine (Figura 3) recibió el Premio Nobel de Química por su teoría termodinámica (entropía) de sistemas alejados del equilibrio (disipativos o no lineales). Al darle un papel protagónico al azar, estableció la imposibilidad de tener certezas absolutas. Demostró que en el mundo hay una creación simultánea de orden y desorden (teoría del caos o de los sistemas dinámicos no lineales). Sentenció: “el caos posibilita la vida y la inteligencia". La teoría del caos se dimensionó significativamente ante el advenimiento de las computadoras. Edward Lorenz (1917-2008), meteorólogo norteamericano, introdujo los conceptos de "efecto mariposa" y "atractor extraño". James A. Yorke (nacido en 1941) aplicó el hallazgo de Lorenz creando una ecuación logística de evolución de una población silvestre, demostrando que "sistemas sencillos son capaces de derivar cosas complejas", principio que luego se aplicó a la genética, economía, dinámica de fluidos, epidemiología y fisiología 7,9,24 .

Benoit Mandelbrot (1975), al comparar la evolución mundial de los precios del algodón en el siglo XX encontró una estructura regular; lo mismo sucedió con las rentas. Para explicar estos fenómenos inventó el término fractal, definido como "autosemejanza de irregularidades a diferentes escalas". Encontró estruc- plia "banda de frecuencias", muy flexibles y adaptables. Dijo "en biología se llega al equilibrio con la muerte". Tom Ray (1989), basado en técnicas de matemática caótica, creó el primer programa computacional de "vida artificial" (simulación biológica), en el cual a partir de reglas de mutación se verificó la evolución de predadores, la aparición de parásitos e incluso extinciones completas y resurgencias. Joseph Ford (1990) efectuó estudios que lo llevaron a postular que "la evolución es caos con realimentación". "El universo se compone de azar y disipación; el azar llega a producir una complejidad asombrosa y la disipación es un agente del orden" 7,9,11.

\section{Nuevos términos}

El ruido, en el lenguaje de la dinámica del caos, es una señal que muestra un movimiento irregular y que no tiene una dimensión finita: en términos fisico-matemáticos, "una señal irregular cuya dimensión de correlación no muestra saturación" 1 .

El espacio de fase es la representación gráfica del comportamiento de un sistema. Existen varias técnicas para elaborarlo, una de ellas consiste en insertar las diferentes variables del sistema en un eje de coordenadas, otra es relacionar una función versus la derivada de dicha función. También se puede representar una función contra sí misma, introduciendo un desfase ${ }^{6}$. En el sistema circulatorio sanguíneo, el espacio de fase se logra graficando unas contra otras las principales variables del sistema (presión contra volumen en el ciclo cardíaco), o bien relacionando una función contra su derivada (curvas del flujo de aire versus volumen durante el ciclo respiratorio) ${ }^{9}$. 
La dimensión es una de las medidas cuantitativas clásicas de un sistema complejo. El mecanismo para investigar la conducta de un sistema es calcular su dimensión. La dimensionalidad de un sistema dinámico es la medida del número de variables independientes que se necesitan para especificar su actividad en un momento dado, lo cual estima la complejidad y los grados de libertad del sistema estudiado ${ }^{1}$. P.ej.: para conocer la variable "clima", se medirán las dimensiones: temperatura, humedad, presión, viento, precipitaciones.

Un atractor es la figura que se genera en el espacio de fase ${ }^{12}$. Los sistemas caóticos son influenciados por "atractores extraños", caracterizados por poseer formas muy variadas con trayectorias impredecibles localmente, pero circunscriptas en un mismo subespacio del espacio de fase (estabilidad global con inestabilidad local). Se considera que un sistema caótico como el organismo en estado de salud coexiste con un atractor extraño: la muerte ${ }^{6,34}$

Los fractales son estructuras complejas que muestran auto-similitud independientemente de la escala con la que se examinen. Entonces, se denomina "fractal" a aquella estructura que consta de fragmentos de orientación y tamaño variable pero de aspecto similar, cuyas características les confieren propiedades geométricas especiales en cuanto a su longitud y relación superficie/volumen, por lo cual su cuantificación requiere herramientas matemáticas diferentes a las convencionales ${ }^{16}$.

\section{Fractales}

Para comprender el funcionamiento de algo, normalmente se tiende a efectuar simplificaciones, p.ej.: al estudiar el movimiento de un cuerpo se obviará la fricción, o al intentar reproducir un paisaje boscoso se utilizarán elementos de la geometría clásica (conos, cilindros, círculos, triángulos). Es claro que los objetos reales son más complicados que las formas geométricas citadas, sin embargo podemos servirnos de ellas para simplificar nuestros intentos de reproducir la realidad. Una manera de hacerlo es utilizar fractales, que son formas geométricas que entre otras propiedades, contienen una imagen de sí mismas en cada una de sus partes. Los fractales han inundado el mundo científico con un conjunto de nuevas reglas útiles para enfrentar el reto de conocer y describir la naturaleza. Su lenguaje se infiltró a campos increíblemente diversos de las ciencias naturales y sociales y ha hecho de las matemáticas un instrumento novedoso para las artes ${ }^{33,35}$.

En biología, la dinámica caótica parece sustentarse en estructuras de carácter fractal, que ostentan mayor redundancia de la información debido a la autosimilitud de estructuras biológicas subyacentes, que operarían aumentando el índice de seguridad en la transmisión de las señales. Por otra parte, los sistemas dinámicos aportan respuestas de mayor flexibilidad, favoreciendo la adaptación a las cambiantes demandas del ambiente, gracias a su especial sensibilidad ante pequeñas modificaciones en las condiciones iniciales. Esta mayor flexibilidad explicaría la variabilidad de las respuestas encontradas en los sistemas y la imposibilidad de su predicción exacta, no por la aleatoreidad de los sistemas, sino por su dinámica caótica ${ }^{1}$.

Muchas cosas en la naturaleza tienen características fractales (objetos porosos, cuerpos rugosos, estructuras que se ramifican). Para medir la superficie de una piedra habrá que aproximarla a la de una esfera o cubo. Para calcular la superficie de absorción del intestino la medida cambiará según la resolución que utilice para hacerlo, debido a que el intestino presenta pliegues desde el nivel macroscópico hasta el microscópico ${ }^{9}$.

En la década de 1970 se estableció que los estudios de pautas irregulares en los procesos naturales y la exploración de sus formas infinitamente complejas, tienen una propiedad común: la cualidad de autosemejanza. Fractal significa sobre todo autosemejanza, término que expresa simetría dentro de una escala, implica recurrencia, pauta en el interior de una pauta ${ }^{16}$.

En la Figura 4 se esquematiza un ejemplo de estructura fractal conocida como "copo de nieve de Koch" (o "estrella de Koch"), una curva cerrada continua pero no diferenciable en ningún punto, descrita por el matemático sueco Helge von Koch en 1904. En lenguaje actual diríamos que es una curva fractal. Su construcción se realiza mediante un proceso iterativo que se inicia con un triángulo equilátero de determinado tamaño. En el centro de cada lado se añade otro triángulo que mida un tercio del original y se procede de esta manera con cada uno de los lados de los nuevos triángulos. El límite de la longitud de la línea exterior final será [ $3 * 4 / 3$ $* 4 / 3 * 4 / 3 \ldots$ infinito ]. Sin embargo, el área resultará menor que la de un círculo trazado alrededor del triángulo primitivo. Por lo tanto "una línea infinitamente larga rodea un área finita" ${ }^{1}$.

\section{Atractores}

Los sistemas dinámicos (deterministas) se clasifican básicamente en estables, inestables y caóticos. A lo largo del tiempo, un sistema estable tiende a ser atraído o repelido hacia o desde un punto u órbita, según su dimensión (atractor o sumidero). Un sistema inestable se escapa de los atractores. Un sistema caótico mani-

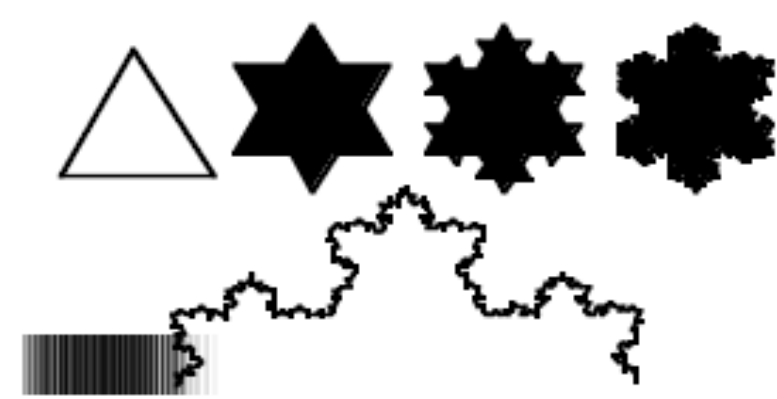

Figura 4. Ejemplo de estructura fractal, producto de la superposición de triángulos reducidos a un tercio del anterior. 
fiesta los dos comportamientos: por un lado, existe un atractor por el cual el sistema es atraído, pero a la vez, hay "fuerzas" que lo alejan de éste. Una de las mayores características de un sistema inestable es que tiene una gran dependencia de las condiciones iniciales. Si estas condiciones iniciales son fijas, conociendo las ecuaciones características de un sistema inestable se puede predecir su evolución en el tiempo. En los sistemas caóticos, como se dijo, una mínima diferencia en esas condiciones hace que el sistema evolucione de manera totalmente distinta, p.ej.: el comportamiento de las placas tectónicas, de los fluidos en régimen turbulento, los crecimientos de población, los movimientos en el sistema solar y muchos otros ${ }^{19}$.

El atractor es la representación geométrica de la dinámica del sistema en el tiempo; los atractores pueden ser caracterizados por sus dimensiones. Un atractor de dimensión 0 corresponde a un sistema estático: el sistema no cambia en el tiempo. Un atractor de dimensión 1 corresponde a un sistema periódico, en el cual un número finito de estados se repiten indefinidamente. Un atractor de dimensión 2 y mayores corresponde a un sistema cuasi-periódico; en el caso de un péndulo oscilante, el atractor sería el punto de equilibrio central. Un atractor periódico puede guiar el movimiento de un péndulo en oscilaciones periódicas; sin embargo, el péndulo puede registrar trayectorias erráticas alrededor de estas oscilaciones debidas a otros factores ${ }^{1,19}$.

Entonces, un atractor es el comportamiento que adopta un sistema ante el influjo de un determinado estímulo. La cuenca de un atractor es el conjunto de condiciones iniciales a partir de las cuales el sistema sigue un determinado comportamiento. La trayectoria es una descripción matemática de la secuencia de valores que toma la variable desde la condición inicial. Los atractores pueden ser periódicos, cuasiperiódicos y caóticos (o atractores extraños) ${ }^{1}$.

\section{Atractores extraños}

Son los que están ligados a los movimientos caóticos y se caracterizan porque pueden llegar a tener una enorme complejidad, como el famoso atractor de Lorenz, derivado del modelo tridimensional de sistema climático del mismo autor (Figura 5). Este atractor es quizás uno de los diagramas de sistemas caóticos más conocidos, no sólo porque fue uno de los primeros, sino también porque es uno de los más complejos y peculiares, pues desenvuelve una forma cuya silueta es parecida a las alas de una mariposa ${ }^{19}$.

La Figura 5 pone en evidencia una estructura que esconde una serie desordenada de datos. Para evidenciar las relaciones mutables entre tres variables, cada punto de la línea representa la situación de las tres variables en un momento dado; por lo tanto cada movimiento del punto representa un cambio en el valor de una o más variables. Como el sistema no se repite de un modo exacto, la trayectoria nunca se corta a sí misma, sino que describe curvas una y otra vez ${ }^{1}$.
Los atractores extraños poseen típicamente estructuras fractales y sólo pueden presentarse como un sistema continuo dinámico si tienen tres o más dimensiones. En esencia, son curvas de los espacios de fases que describen la trayectoria elíptica de un sistema en movimiento caótico. Un sistema de estas características es totalmente impredecible; conocer la configuración del sistema en un momento dado no permite predecir con veracidad su configuración en un momento posterior. De todos modos, los movimientos caóticos no son completamente aleatorios; en la mayoría de los sistemas dinámicos se encuentran elementos que permiten un tipo de movimiento repetitivo, a veces geométricamente establecido. Los atractores son los encargados de que las variables que se inician en un punto de partida mantengan una trayectoria establecida. Lo que no se puede establecer de manera precisa son las oscilaciones que las variables puedan tener al recorrer las órbitas que puedan llegar a establecer los atractores ${ }^{19}$.

\section{Lógica difusa}

La lógica del caos, difusa o borrosa es un sistema matemático que modela funciones no-lineales acordes a los planteamientos lógicos que usan el "razonamiento aproximado". Se fundamenta en los denominados conjuntos difusos y en un sistema de inferencias borrosas basado en reglas de la forma relativa ("si... entonces...”), donde los valores lingüísticos de la premisa y el consecuente están definidos por conjuntos borrosos. Así, esta lógica se basa en la relatividad de lo observado. Veamos un ejemplo "fisiológico" de abordaje:

Juan mide $2 \mathrm{~m}$ y es diestro, María mide $1.65 \mathrm{~m}$ y tiene ojos azules, Pedro es zurdo de ojos marrones, luego ¿será alto o bajo?

La lógica lineal se desarticula al introducir una incertidumbre inesperada o incongruente con los postulados iniciales. La respuesta surgirá de los cálculos realizados por modelos informáticos capaces de valorar la incertidumbre. Científicamente, la incertidumbre

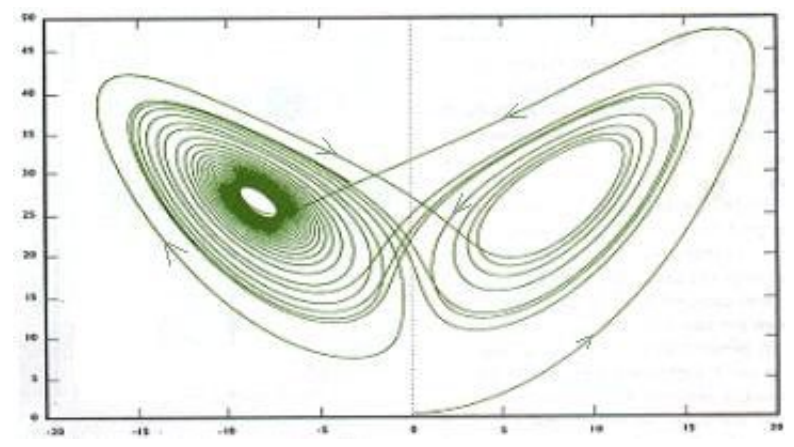

Figura 5. Atractor de Lorenz. Figura geométrica similar a las alas de una mariposa donde los cambios de dirección de la línea representan cambios del valor de las variables. 
es "una estimación unida al resultado de un ensayo que caracteriza el intervalo de valores dentro de los cuales se afirma que está el valor verdadero". Las situaciones de incertidumbre son recurrentes, su periodicidad responde a una repetición (frecuencia) abordable por las estadísticas ${ }^{36}$.

Los primeros éxitos de la lógica del caos ocurrieron en el campo del control automático de los procesos, especialmente en Japón a principios de la década de 1990. Ya en 1974 Mamdani había experimentado con éxito un controlador borroso en una máquina de vapor; en 1983 Fuji aplica lógica difusa para el control de inyección química en instalaciones depuradoras de agua. Luego esta lógica se aplicó en diversas ramas tecnológicas como metalurgia, robots, sensores de imagen y sonido (Sony, Sanyo, Cannon), electrodomésticos (Panasonic, Bosch), aire acondicionado (Mitsubishi), sistema de frenado antibloqueo ABS (Mazda, Nissan), cajas de cambio automático (Renault) y controles de maniobra de aviones, ascensores y trenes de alta velocidad (el tren-metro de Sendai, Japón, entre otros) ${ }^{36}$.

\section{Matemática del caos}

La teoría del caos indujo la aparición de la matemática caótica, la cual resultó ser una herramienta aplicable a muchos campos de la ciencia y la tecnología. Gracias a estas aplicaciones el nombre se torna paradójico, dado que muchas de las prácticas que se realizan con la matemática caótica tienen resultados concretos porque los sistemas que se estudian están basados estrictamente en leyes deterministas aplicadas a sistemas dinámicos. La estadística inferencial trabaja con modelos aleatorios para crear series caóticas predictoras de eventos presumiblemente caóticos. Por esta razón la teoría del caos ya no es en sí una teoría: tiene postulados, fórmulas y parámetros recientemente establecidos con aplicaciones en áreas de fisiología, medicina, física, meteorología, economía y otras ${ }^{19}$.

La mecánica cuántica maneja magnitudes observables, ciertas e inciertas, que no siempre varían de una manera continua y donde las probabilidades asociadas a cada valor provienen de un azar objetivo. La incertidumbre o indefinición no proviene de la ignorancia, por lo cual es una incertidumbre probabilizable que puede ser estimada mediante inferencia estadística. En el nuevo paradigma de la complejidad, el caos nos conduce a nuevos modelos que dan respuesta a esas inestabilidades, para las cuales el reduccionismo neoclásico ni siquiera admitía preguntas. En la modelización matemática la noción de trayectoria es fundamental y la predicción es exacta, cualquiera sea el tiempo. En la modelización estadística, reductible a trayectorias, a medida que el tiempo de predicción se alarga, el intervalo de predicción se hace más amplio ${ }^{21}$.

Poniendo en conexión esta evolución con una ecuación no-lineal (logística) que atraviese pequeñas inestabilidades, surge que el incremento de incertidumbre puede ser compensado con información y con peque- ños cambios desde el entorno, pudiendo ser aplicada una estadística dinámica (lineal) hasta que, ante mayores inestabilidades, serán influenciadas por la aparición de desorden y fractalidad (dinámica caótica), en correspondencia con la historia de un sistema que, en contacto con las nuevas realidades y valores de su entorno "siente la llama seductora del atractor caótico" 21.

El mundo está lleno de fenómenos que parecen caóticos aunque en realidad están ceñidos a reglas estrictas pero difíciles de esclarecer por la gran cantidad de variables implicadas o de combinaciones generadas, solo desentrañables con una herramienta aparecida recientemente en la ciencia, la computación. Con ella se demostró que las cosas no ocurren al azar, porque están determinadas por las condiciones iniciales. La dinámica y complejidad de tales condiciones torna casi impredecible el efecto ${ }^{7}$.

Los modelos numéricos surgidos de la teoría del caos han introducido considerables mejoras en la exactitud de las previsiones meteorológicas en comparación con las predicciones anteriores, especialmente para períodos superiores a un día. Actualmente es posible demostrar la confiabilidad de las predicciones específicas para períodos de hasta 5 días gracias a la densidad entre las órbitas periódicas del sistema, y se han logrado algunos éxitos en la predicción de variaciones anormales de la temperatura y la pluviosidad para periodos de hasta 30 días ${ }^{13-15,22}$.

\section{Seres vivos y caos}

La teoría del caos y los fractales son valiosas herramientas para el análisis y comprensión del modelo biológico, a través de una transformación epistémica significativa del conocimiento científico. Sustituyendo al reduccionismo y la simplificación, el paradigma de la complejidad se instaló definitivamente a través del enfoque multidimensional y multicausal. Los seres vivos son sistemas hipercomplejos y adaptativos porque toman datos de su funcionamiento interno y del ambiente que los rodea para automodificar algunas de sus características, p.ej. para adaptarse a los cambios o para responder a la agresión de la enfermedad. Así, los organismos son sistemas caóticos, no operan al azar, son deterministas porque hay algo que determina su comportamiento, son muy sensitivos a las condiciones iniciales y parecen desordenados pero no lo son: hay reglas que determinan su comportamiento. A pesar de las muy diferentes condiciones externas a que puede estar expuesto (clima, estrés, esfuerzo físico), siempre mantiene una integridad general. Tal integridad debe ser conservada cuando una enfermedad agrede las condiciones internas del sistema, lo cual demandará un cambio en la estructura. El corazón ejemplifica el vigor del sistema caótico, al desarrollar un abanico de comportamientos ("grados de libertad") que incluyen profundos cambios en su ritmo y fuerza de contracción, para luego retornar a su actividad habitual ${ }^{6}$. 
El organismo viviente es un sistema complejo adaptable a variaciones externas, cuya supervivencia se debe a un conjunto de fenómenos que se desarrollan de manera impredecible dentro de sus altos niveles de organización funcional jerárquica, de comportamiento a-periódico muy sensible a las condiciones iniciales, con recurrencia de ciertos patrones a diferentes escalas espaciales y temporales ${ }^{6,24}$.

\section{Caos y fisiología}

Según la teoría del caos, la fisiología y la patología ya no pueden ser consideradas como fenómenos exclusivamente biológicos y los recursos terapéuticos no pueden limitarse solamente a la cirugía o farmacología. Desde la teoría del caos se postula la sustitución del reduccionismo y la simplificación, por un pensamiento complejo y transdisciplinar que plantee los fenómenos biológicos como heterogéneos y multicausales, creando así modelos más apropiados para su estudio ${ }^{4,6}$.

Se postula que la dinámica fisiológica es explicada por estructuras fractales, como las del sistema nervioso, las ramificaciones de los tubos bronquiales en el pulmón, la circulación de los fluidos corporales, las variaciones hormonales y las fibras del sistema excito-conductor del corazón, que permiten una amplia serie de ritmos. Todos ellos son sistemas dinámicos caóticos disipativos de energía. Los ritmos biológicos podrían ser interpretados como la sincronización de la dinámica de estructuras fractales que reproducen un modelo escalar. Las estructuras fractales asociadas con espectros escalares y de banda amplia son ricos en información. En cambio, los estados periódicos reflejan espectros de banda estrecha, cuyas secuencias monótonas y reiterativas están vacías de contenido informador. Con esta óptica, algunas enfermedades podrían ser tratadas ampliando la reserva espectral de un sistema, de tal manera que pueda recorrer muchas frecuencias sin caer en un canal cerrado periódico ${ }^{1,12}$.

Los seres vivos poseen biorritmos que fluctúan de un modo complejo y aparentemente aleatorio (variaciones circadianas, interacciones hormonales e inmunoló-

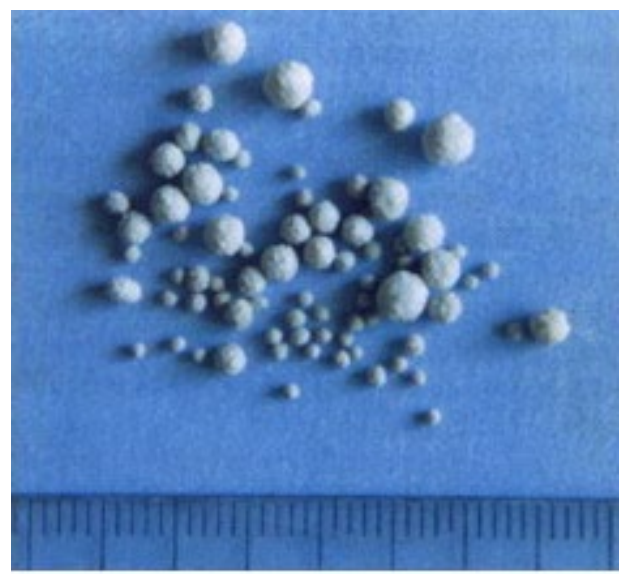

Figura 6. Urolitos de urato de amonio extraídos de la vejiga urinaria de un perro. gicas, ondas electroencefalográficas y electrocardiográficas), discutiéndose en términos fisico-matemáticos si en ellos subyace un caos determinista o simplemente ruidos aleatorios. Actualmente tiende a admitirse que la mayoría de los biorritmos son caóticos, aunque no exentos de un cierto factor de ruido aleatorio ${ }^{32}$

Según la homeostasis, un organismo estabilizará su ritmo cardíaco cuando cesen las perturbaciones circunstanciales que lo alejaron de su estatus fisiológico. Sin embargo, recientes evidencias demostrarían que aún en ausencia total de estímulos perturbadores, el corazón es intrínsecamente caótico, su ritmo es siempre irregular y posee un atractor extraño cuyo análisis matemático permite realizar pronósticos que estarían fuera del alcance de la semiología cardiológica clásica 12,32 .

Siguiendo en el campo de la fisiología, el cerebro es otro órgano que genera ondas con un atractor extraño subyacente, cuya actividad puede ser caracterizada para estudiar determinadas enfermedades mentales o la propensión a padecerlas. La inmunidad es un delicado y complejo sistema dinámico caótico que puede oscilar en un "ciclo límite" en respuesta a determinadas "perturbaciones" como enfermedades mieloproliferativas. Las actividades enzimáticas y las retroacciones hormonales también están sujetas a la dinámica caótica 11, 12, 17 . Algunos investigadores han propuesto modelos caóticos determinísticos para desentrañar algunos aspectos del cáncer ${ }^{23}$.

En fisiología pueden distinguirse estructuras fractales como la red vascular, el árbol bronquial, la trama neuronal, la mucosa intestinal y otras. Las estructuras fractales optimizan la función de los sistemas al poseer una gran superficie con muy poco volumen. Los pulmones p.ej. tienen un área de intercambio de aproximadamente $150 \mathrm{~m}^{2}$, mientras que su volumen total es de unos 7-8 litros ${ }^{4}$.

Un estudio fisiológico en ratones demostró que para la percepción olfativa, el cerebro dirige al sujeto hacia la búsqueda de información mediante una actividad autoorganizadora que se desarrolla en el sistema límbico; éste libera un mensaje de re-aferencia, alertando a los sistemas sensoriales a prepararse para responder a nueva información. Las redes neuronales responderían en ráfagas. La actividad sincrónica de cada sistema se retransmite hasta el sistema límbico, donde se combina con la salida producida de manera similar por los otros sistemas, formando una "gelstat" (un todo mayor que la suma de sus partes). El atractor sería el influjo de un determinado estímulo capaz de generar cambio de conducta. La función integrativa del sistema nervioso central sería una buena aproximación al concepto de atractor ${ }^{10}$.

Un sistema caótico está diseñado y construido para asegurar su propia estabilidad y controlar fuentes de "ruido", mediante un mecanismo extremadamente (aunque no absolutamente) estable. La auto-organización neural hace necesaria una reevaluación de la utilización de modelos informáticos digitales y analógicos 
en las ciencias cognitivas: sin la conducta caótica el sistema neural no podría añadir conocimientos nuevos. El caos provee al sistema un determinístico estado de "no se" dentro del cual se genera un nuevo patrón de actividad. Tanto el electroencefalograma (EEG) como la cognición son actividades que comparten algunas características que las hacen isomórficas: ambas son continuas en el tiempo, evolucionan con la maduración y la edad, responden a estimulación tanto interna como externa y son autoorganizadas ${ }^{30}$.

La aplicación de la dinámica caótica a la conducta constituye un prometedor desarrollo teórico, porque implica que los sistemas biológicos son abiertos (intercambian energía con el ambiente), no-lineales (gobernados por ecuaciones diferenciales no-lineales o de diferencia) y autoorganizados. La rápida pérdida de predictibilidad de estos sistemas caóticos no sería debida a su aleatoriedad sino a la extremada sensibilidad a condiciones iniciales del sistema, por la cual pequeñas diferencias en el estado de un sistema resultarían en una divergencia exponencial en su conducta futura ${ }^{30}$.

El EEG resulta de la suma de los potenciales postsinápticos originados en la corteza cerebral y refleja la actividad eléctrica de aproximadamente un billón de neuronas. Diversos trabajos han demostrado que la actividad eléctrica cerebral es influenciada por atractores extraños, por lo cual el comportamiento del EEG debe ser considerado caótico ${ }^{2,3,25,27}$.

Por su parte, otros investigadores asumen que el caos puede tener un importante papel en la generación de una adaptación rápida (nerviosa) a los cambios ambientales de un organismo. Las señales que no varíen, como las de ciclos limitados, no llevarán nueva información al futuro que no sea la que ya contenían. Trabajos sobre la respuesta de potenciales evocados en animales y seres humanos apoyan esta opinión, mostrando cómo los potenciales están reducidos o desaparecen si una estructura cerebral muestra actividad de ciclo limitado, p.ej. la actividad regular theta del hipocampo durante la cual no se encuentran potenciales evocados de gran amplitud. También se asume que la evolución del caos a largo plazo es impredecible y que tal impredectibilidad representa una ganancia de información por la cual el cerebro crea nuevas respuestas posibles ${ }^{20}$.

\section{Caos y patología}

En patología, la teoría de los fractales fue aplicada exitosamente para predecir la aparición de osteoporosis a partir de un programa informático que comparaba la textura de los huesos de un individuo versus patrones anormales ${ }^{4}$. A manera de "efecto mariposa", el cambio de solamente un aminoácido por otro en una proteína compuesta por miles de aminoácidos, p.ej. la que constituye el canal de potasio de la membrana celular de la fibra muscular del ratón, acarrea graves consecuencias como la ataxia motora del roedor ("canalopatía"). En otra proteína como la cadena beta de la hemoglobina humana, la sustitución de un solo aminoácido cons- titutivo (glutamina) por otro foráneo (valina), genera fragilidad y ruptura de los eritrocitos configurando la denominada "anemia drepanocítica o falciforme". En la orina, las moléculas de solutos se mueven desordenadamente (caos) por su energía libre (cinética molecular); si se reduce tal energía libre (ordenamiento del caos), el movimiento cesa y las moléculas se agrupan formando cristales que a su vez pueden generar cálculos en vías urinarias (Figura 6) ${ }^{6,12,22}$.

Es conocido que los pacientes esquizofrénicos revelan trastornos de la motilidad del globo ocular: ellos no logran seguir el suave movimiento de un péndulo que oscila lentamente ante sus ojos, sino que éstos saltan de forma desorganizada sin llegar a posarse en el blanco. Un físico llamado Huberman preparó un modelo explicativo de este movimiento consistente en una ecuación que analogaba un sistema mecánico donde una pelota giraba en un recipiente curvado que se balanceaba con movimiento pendular. Al aumentar el poder de no-linealidad, el sistema se desordenaba caóticamente tal como lo hacían los ojos de los pacientes esquizofrénicos. Muchos se preguntaron ¿será posible que la inestabilidad sea salud y la estabilidad (caos) una enfermedad? Desde la teoría del caos debería responderse afirmativamente. La dinámica del EEG en patologías como la esquizofrenia o la epilepsia genera atractores cerrados de bajo valor dimensional, que resultan muy estables. Tal estabilidad disminuye las posibilidades de "cambio" o "bifurcación", ensombreciendo el cuadro. La posibilidad de introducir bifurcaciones en estos sistemas podría mejorar el pronóstico ${ }^{1}$. La gran ventaja de los sistemas caóticos determinísticos es que permiten cambiar la actividad casi instantáneamente a través de bifurcaciones, o sea súbitas transiciones desde una actividad de ciclo limitado a una actividad caótica, como la observada en muchos sistemas cuando el valor de un parámetro es ligeramente alterado ${ }^{29}$.

Se piensa que los agentes causantes de crisis epilépticas del tipo "petit mal" tienden a conducir la actividad cerebral hacia un movimiento periódico estable. Superar tal estado sería extraordinariamente difícil y sólo posible mediante la dinámica caótica de la actividad cerebral. El EEG de un cerebro sano es irregular; esta actividad experimenta un cambio drástico con el inicio de un acceso epiléptico: la actividad eléctrica es más violenta en términos de amplitud, pero más regular rítmicamente ${ }^{2}$.

La presencia de actividad caótica en un punto dado del registro EEG no garantiza que el caos esté presente simultáneamente en otras áreas del cerebro. De hecho, existe evidencia de que los lóbulos frontales pueden generar ruido durante largos períodos, al mismo tiempo que los occipitales muestran conducta caótica. Se ha propuesto que el caos surge en el cerebro cuando dos o más áreas cumplen al menos dos condiciones: se excitan una a otra con suficiente fuerza como para impedir que cualquiera de ellas quede en reposo y, al mismo tiempo, son incapaces de coincidir en una frecuencia común oscilatoria, contribuyendo al aumento de la sen- 
sibilidad y la inestabilidad del sistema, al caos. En una investigación se encontró incremento de la actividad EEG en la banda delta en pacientes esquizofrénicos, con mayor grado de complejidad dimensional en áreas frontales que en áreas centrales, a la inversa de lo registrado en individuos sanos ${ }^{8}$.

Estudios realizados usando caos determinístico revelaron mayores dimensiones en las condiciones de EEG de vigilia que en el EEG de sueño y epilepsia. Bajas dimensionalidades fueron encontradas entre EEG de reposo y ojos cerrados y mayores en situaciones de actividad cognitiva. El hecho que en el reposo el atractor sea fractal y disminuya con la profundidad del sueño, implica que la dinámica del sueño profundo llega a ser más coherente durante estas etapas ${ }^{2,26}$.

La actividad caótica de baja dimensión y alta sincronía (ritmicidad) fue verificada en patologías como demencia, esquizofrenia y epilepsia, en contraste con sujetos sanos que presentaron una actividad EEG de mayor dimensionalidad y mayor variabilidad de la señal (menor ritmicidad). Puesto que la actividad eléctrica cerebral comparte importantes características con la actividad cognitiva (secuencialidad, continuidad, evolución, sensibilidad a señales internas y externas y autoorganización) resulta posible que también la propia actividad cognitiva esté expuesta a la dinámica caótica no aleatoria ${ }^{26}$.

\section{Conclusión}

La aparición de la teoría de caos ha generado un cambio de paradigma en la filosofía de la ciencia, posibilitando encontrar el orden en el desorden. Sustituyendo al reduccionismo, el caos determinístico instaló un enfoque complejo, multicausal y multidimensional que involucra una realidad como un todo mayor a la suma de sus partes, donde hechos insignificantes pueden afectar el comportamiento y la evolución de la naturaleza. Más que una teoría, el caos se ha convertido en un método, un modelo, una forma de generar conocimientos científicos en el área de los sistemas complejos.

\section{Referencias}

1. Andreu C, Echave JD, Buela-Casal G. 1998. Actividad electroencefálica según la teoría del caos. Psicothema (Zaragoza) 10: 319-331.

2. Babloyantz A, Destxhe A. 1986. Low dimensional chaos in an instance of epilepsy. Nat Acad Sci USA 83: 35133517.

3. Basar E. 1990. Chaos in brain function, Springer-Verlag, New York, p. 1-30.

4. Bassingthwaighte L, Liebovitch L, West B. 1994. Fractal physiology, Oxford Univ Press, Oxford, 364 p.

5. Braun E. 1996. Caos, fractales y cosas raras, Ed. Fondo Cult Econ, México, 154 p.

6. Chávez-Grimaldi OM. 2006. La enfermedad: una visión desde la teoría del caos y de los fractales. Rev Med Int Crit (Valencia) 3: 78-84.
7. Colle R. 2002. La computación desentraña el caos y la complejidad. Publicación on line: http://www2.ing.puc. $\mathrm{cl} / \sim$ dcolle/publicaciones/caos/caos.htm.

8. Elbert T, Lutzenberger W, Rockstroh B, Berg P, Cohen P. 1992. Physical aspects of the EEG in schizophrenics. Biol Psych 32: 595-606.

9. Farbiarz FJ, Alvarez DL. 2010. Complejidad, caos y sistemas biológicos. Publ. on line: http://www.encolombia. com/medicina/academedicina/m-02JFarbiarz.htm.

10. Freeman WJ. 1991. The physiology of perception. Scie Am 264: 78-85.

11. Gleick J. 1988. Caos. La creación de una ciencia, Ed. Seix Barral, Barcelona, $358 \mathrm{p}$.

12. Goldberger AL. 1996. Non-linear dynamics for clinicians. Chaos theory, fractals and complexity at the bedside. Lancet 347: 1312:1314.

13. González-Miranda JM. 2004. Synchronization and control of chaos. An introduction for scientists and engineers, Imperial College Press, London, $292 \mathrm{p}$.

14. Gutzwiller M. 1990. Chaos in classical and quantum mechanics, Ed. Springer-Verlag, New York, 412 p.

15. Hoover WG. 2001. Time reversibility, computer simulation, and chaos, Ed. World Scientific, Singapore, 367 p.

16. Mandelbrot BB. 1997. La geometría fractal de la naturaleza, Ed. Tusquets, Barcelona, 442 p.

17. May RM. 1976. Simple mathematical models with very complicated dynamics. Nature 261: 459-476.

18. Montero ML. 2010. Teoría del caos. Publicación on line: http://marialuisamontero. blogspot.com /2010/04/teoriadel-caos.html.

19. Moon F. 1990. Chaotic and fractal dynamics, SpringerVerlag, New York, 355 p.

20. Mpitsos GJ, Cohan CS. 1986. Convergence in a distributed nervous system: parallel processing and self-organization. J Neurobiol 17: 517-545.

21. Nieto UA. 2009. Estabilidad, caos y crisis financiera. Publicación on line: http://www.actuarios.org /espa/anales/2009/Pag\%2071-100.pdf.

22. Ott E. 2002. Chaos in dynamical systems, Cambr Univ Press, Cambridge, $492 \mathrm{p}$.

23. Patiño RJ. 2002. El cáncer desde la perspectiva de la teoría del caos. Rev Colomb Cirug (Bogotá), 17: 3-5.

24. Prigogine I. 1993. ¿Tan Sólo Una Ilusión? Una exploración del caos al orden, $3^{\circ}$ ed., Ed. Tusquets, Barcelona, p. 159-160.

25. Rapp PE, Bashore TR, Martinerie JM, Albano AM, Mees AI. 1989. Dynamics of brain electrical activity. Brain Topogr 2: 99-118.

26. Renshaw E. 1994. Chaos in biometry. J Math App Med Biol 11: 17-44.

27. Röschke J. 1992. Strange attractors, chaotic behavior and informational aspects of sleep EEG data. Pharmacoelectroencephalography 25: 172-176.

28. Schifter I. 1996. La ciencia del caos, Ed Fondo Cult Econ, México, $106 \mathrm{p}$.

29. Serra R, Zanarini G. 1990. Complex systems and cognitive processes, Springer-Verlag, New York, $415 \mathrm{p}$. 
30. Skarda A, Freeman WJ. 1987. How brains make chaos in order to make sense of the world. Behav Brain Sci 10: 161-195.

31. Skinner JE, Molnár M, Vybirar T, Mitra M. 1992. Application of chaos theory to biology and medicine. Integr Physiol Behav Sci 27: 39-53.

32. Subias JL. 1992. Aplicaciones de la teoría del caos en medicina. Publicación on line: http://www.didyf.unizar.es/ info/jlsubias/Cor_tv04.htm.
33. Talanquer V. 1996. Fractales, de laberintos y espejos, Fondo Cult Econ, México, 108 p.

34. Trilla A. 1998. El caos y la predicción médica. Med Clin Barc 110: 619-620.

35. Wegner T, Tyler B. 1995. El mundo de los fractales, Ed. Anaya Multimedia, Madrid, $412 \mathrm{p}$.

36. Zadeh LA, Desoer CA. 2008. Linear system theory: the state space approach, Dover Publications, New York, 656 p. 